\title{
Models for Multi-Specie Chemical Reactions Using Polynomial Stochastic Hybrid Systems
}

\author{
Abhyudai Singh and João Pedro Hespanha
}

\begin{abstract}
A procedure for constructing approximate stochastic models for chemical reactions is presented. This is done by representing the population of various species involved in a chemical reaction as the continuous state of a polynomial Stochastic Hybrid System (pSHS). An important property of pSHSs is that the dynamics of all the statistical moments of its continuous states, evolves according to a infinite-dimensional linear ordinary differential equation (ODE). Under appropriate conditions, this infinite-dimensional ODE can be accurately approximated by a finite-dimensional nonlinear ODE, the state of which typically contains the moments of interest. In this paper, for a very general class of chemical reactions, we provide existence and uniqueness conditions for these finite-dimensional nonlinear ODEs. Furthermore, explicit formulas to construct them are also provided.
\end{abstract}

To illustrate the applicability of our results, we construct an approximate stochastic model for a decaying and dimerizing chemical reaction set. Moment estimates obtained from the finite-dimensional nonlinear ODE are compared with estimates obtained from a large number of Monte Carlo simulations.

\section{INTRODUCTION}

The time evolution of a spatially homogeneous mixture of chemically reacting molecules is often modeled using a stochastic formulation, which takes into account the inherent randomness of thermal molecular motion. This formulation is superior to the traditional deterministic formulation of chemical kinetics and is motivated by complex reactions inside living cells, where small populations of key reactants can set the stage for significant stochastic effects [1]- [4].

In the stochastic formulation, the time evolution of the system is described by a single equation for a grand probability function, where time and species populations appear as independent variables, called the Master equation [5]. However, this equation can only be solved for relatively few, highly idealized cases and generally Monte Carlo simulation techniques are used [6]- [9]. Since one is often interested in only the first and second order moments for the number of molecules of the different species involved, much effort can be saved by applying approximate methods to produce these low-order moments, without actually having to solve for the probability density function. Various such

This material is based upon work supported by the Institute for Collaborative Biotechnologies through grant DAAD19-03-D-0004 from the U.S. Army Research Office and by the National Science Foundation under Grant No. CCR-0311084.

A.Singh and J.P.Hespanha are with the Center for Control Engineering and Computation University of California, Santa Barbara, CA 93101. abhi@engineering.ucsb.edu, hespanhadece.ucsb.edu approximate methods have been developed, for example, using the Fokker-Plank approximation or expanding the Master equation [5].

In [10], an alternative approximate method for estimating lower-order moments was introduced. This was done by representing the dynamics of a chemical reaction as a Stochastic Hybrid System (SHS) whose state $\mathbf{x}$, is the population of all the species involved in the reaction. In order to fit the framework of our problem, this class of SHS had trivial continuous dynamics $\dot{\mathbf{x}}=0$, with reset maps and transitional intensities defined by the stoichiometry and the reaction rates of the reactions, respectively. In essence, if no reaction takes place, the population of species remain constant and whenever the reaction takes place, the reset map is "activated" and the population is reset according to the stoichiometry of the reaction. Details for the stochastic modeling of chemical reactions are presented in Sec. II.

It was also shown in [10] that these SHSs used to model chemical reactions are actually polynomial Stochastic Hybrid Systems (pSHS). An important property of pSHSs is that, if one creates an infinite vector containing all the statistical moments of $\mathbf{x}$, the dynamics of this vector is governed by an infinite-dimensional linear ordinary differential equation (ODE) which we call the infinite-dimensional moment dynamics. In general, the infinite-dimensional moment dynamics cannot be solved analytically, however, as shown in Sec. III, under appropriate conditions, they can be approximated by a finite-dimensional nonlinear ODE, which we call the truncated moment dynamics. The state of the truncated moment dynamics $\mu$, typically contains the lower-order moments of interest. A procedure to construct these truncated moment dynamics was outlined in [10].

The procedure proposed in [10] was general but not systematic. Moreover, [10] provided no conditions under which the truncated moment dynamics could be found. In this paper we resolve these issues for a very general class of $K$ chemical reactions involving $n$ species. More specifically, we show in Sec. IV, that given a vector $\mu$, containing all the first and second order moments of $\mathbf{x}$, one can find truncated moment dynamics, if one drops some second and all first order moments from the second time derivative of $\mu$. This will be valid, as long as these moments are dominated by the fourth order moments of $\mathbf{x}$, which also appear in the second time derivative of $\mu$. We also give specific conditions on the stoichiometry of the reactions, for which, 
this truncated moment dynamics is unique. Furthermore, explicit formulas to construct them are also provided. The striking features of these formulas is that are independent of $K$, the stoichiometry of the reactions and the reaction rates.

To summarize, the results of this paper provide a fully automated procedure for constructing truncated moment dynamics for general sets of chemical reactions. To illustrate the applicability of our results we consider a decayingdimerizing reaction set [7], [14]. This reaction is difficult to simulate due to the existence of two very distinct time scales and methods that do not require Monte Carlo simulations are of special interest. Moment estimates obtained from the truncated moment dynamics are compared with estimates obtained from a large number of Monte Carlo simulations.

\section{Stochastic Modeling of Chemically Reacting SYSTEMS}

Consider a system of $n$ species $X_{j}, \forall j=\{1, \ldots, n\}$ inside a fixed volume $V$ involved in $K$ reactions of the form

$R_{i}: u_{a i} A_{i}+u_{i 1} X_{1}+\ldots+u_{i n} X_{n} \stackrel{c_{i}}{\longrightarrow} v_{i 1} X_{1}+\ldots+v_{i n} X_{n}+*$

for all $i \in\{1, \ldots, K\}$, where species $A_{i}$ have a constant number of molecules ${ }^{1}, u_{i j} \in \mathbb{N}_{\geq 0}$ is the stoichiometry associated with the $j^{t h}$ reactant in the $i^{t h}$ reaction and $v_{i j} \in \mathbb{N}_{\geq 0}$ is the stoichiometry associated with the $j^{\text {th }}$ product in the $i^{\text {th }}$ reaction, and $*$ represents products other than the species $X_{j}$. As all chemical reactions occur in a series of elementary reactions [11], which are generally uni- or bi-molecular, we assume

$$
u_{a i}+u_{i 1}+\ldots+u_{i n} \leq 2, \quad \forall i=\{1, \ldots, K\} .
$$

The reaction parameter $c_{i}$ characterizes the reaction $R_{i}$ and, together with the stoichiometry, defines the probability that a particular reaction takes place in an "infinitesimal" time interval $(t, t+d t]$. This probability is given by the product of the following two terms:

1) the number $h_{i}$ of distinct molecular reactant combinations present in $V$ at time $t$ for the reaction $R_{i}$,

2) the probability $c_{i} d t$ that a particular combination of $R_{i}$ reactant molecules will actually react on $(t, t+d t]$.

For simplicity, we assume $c_{i}$ to be constants, but in general they can be allowed to be functions of time. The number, $h_{i}$ depends both on the reactants stoichiometry $u_{i j}$ in $R_{i}$ and on the number of reactant molecules in $V$. Table I shows the value of $h_{i}$ for different reaction types [6]. In this table and in the sequel, we denote by $\mathbf{x}_{j}$, the number of molecules of the species $X_{j}$ in the volume $V$. The reaction parameter $c_{i}$ is related to the reaction rate $k_{i}$ in the deterministic formulation of chemical kinetics by the formulas shown in the right-most column of Table I. For convenience, we group the reactions in (1) into $S$ groups, such that, reactions in each group have

\footnotetext{
${ }^{1}$ We assume that the population of species $A_{i}$ is large compared to species $X_{j}$, and hence, can be assumed to be a constant.
}

TABLE I

$h_{i}(\mathbf{x})$ AND $c_{i}$ FOR DIFFERENT REACTION TYPES

\begin{tabular}{lcc}
\hline Reaction $\mathrm{R}_{\mathrm{i}}$ & $h_{i}(\mathbf{x})$ & $c_{i}$ \\
\hline$X_{j} \longrightarrow *$ & $\mathbf{x}_{j}$ & $k_{i}$ \\
$X_{j}+X_{t} \longrightarrow *, \quad(t \neq j)$ & $\mathbf{x}_{j} \mathbf{x}_{t}$ & $\frac{k_{i}}{V}$ \\
$2 X_{j} \longrightarrow *$ & $\frac{1}{2} \mathbf{x}_{j}\left(\mathbf{x}_{j}-1\right)$ & $\frac{2 k_{i}}{V}$ \\
\hline
\end{tabular}

identical $h_{i}(\mathbf{x})$. Towards that end, we order the reactions in the following way

$$
\begin{aligned}
& \forall i=\left\{K_{0}+1, \ldots, K_{1}\right\}, \quad h_{i}(\mathbf{x})=h_{K_{1}}(\mathbf{x}) \\
& \forall i=\left\{K_{1}+1, \ldots, K_{2}\right\}, \quad h_{i}(\mathbf{x})=h_{K_{2}}(\mathbf{x}) \\
& \quad \vdots \\
& \forall i=\left\{K_{S-1}+1, \ldots, K_{S}\right\}, \quad h_{i}(\mathbf{x})=h_{K_{S}}(\mathbf{x}),
\end{aligned}
$$

where $S \leq K, K_{0}=0$ and $K_{S}=K$. If all the reactions in (1) have different $h_{i}(\mathbf{x})$, then $S=K$ and $K_{i}=i, \forall i=\{1, \ldots, K\}$.

To model the time evolution of the number of molecules $\mathbf{x}_{1}, \mathbf{x}_{2}, \ldots, \mathbf{x}_{n}$, a special class of Stochastic Hybrid Systems (SHS) were introduced in [10]. More specifically, to fit the framework of our problem, these system are characterized by trivial dynamics

$$
\dot{\mathbf{x}}=0, \quad \mathbf{x}=\left[\mathbf{x}_{1}, \ldots, \mathbf{x}_{n}\right]^{T}
$$

a family of $K$ reset maps

$$
\mathbf{x}=\phi_{i}\left(\mathbf{x}^{-}\right), \quad \phi_{i}: \mathbb{R}^{n} \rightarrow \mathbb{R}^{n},
$$

and a corresponding family of $K$ transition intensities

$$
\lambda_{i}(\mathbf{x}), \quad \lambda_{i}: \mathbb{R}^{n} \rightarrow[0, \infty)
$$

for all $i=\{1, \ldots, K\}$. Each of the reset maps $\phi_{i}(\mathbf{x})$, and corresponding transition intensities $\lambda_{i}(\mathbf{x})$ are uniquely defined by the $i^{\text {th }}$ reaction in (1) and given by

$$
\mathbf{x} \mapsto \phi_{i}(\mathbf{x})=\left[\begin{array}{c}
\mathbf{x}_{1}-u_{i 1}+v_{i 1} \\
\mathbf{x}_{2}-u_{i 2}+v_{i 2} \\
\vdots \\
\mathbf{x}_{n}-u_{i n}+v_{i n}
\end{array}\right], \lambda_{i}(\mathbf{x})=c_{i} h_{i}(\mathbf{x})
$$

for all $i=\{1, \ldots, K\}$. In essence, if no reaction takes place, the state remains constant and whenever the $i^{\text {th }}$ reaction takes place, $\phi_{i}(\mathbf{x})$ is "activated" and the state $\mathbf{x}$ is reset according to (7), furthermore, the probability of the activation taking place in an "infinitesimal" time interval $(t, t+d t]$ is $\lambda_{i}(\mathbf{x}) d t$.

\section{MOMEnT DynAmiCS}

To fully characterize the dynamics of a chemical reaction one would like to determine the evolution of the probability distribution for $\mathbf{x}(t)$. In general, this is difficult and a more reasonable goal is to determine the evolution of a few loworder moments.

Given a vector $m=\left(m_{1}, m_{2}, \ldots, m_{n}\right) \in \mathbb{N}_{\geq 0}^{n}$ of $n$ greater than equal to zero integers, we define the test-function 
associated with $m$ to be $\psi^{(m)}(\mathbf{x})=\mathbf{x}^{(m)}, \forall \mathbf{x} \in \mathbb{R}^{n}$ and the (uncentered) moment associated with $m$ to be

$$
\mu^{(m)}(t)=\mathbf{E}\left[\psi^{(m)}(\mathbf{x}(t))\right], \quad \forall t \geq 0 .
$$

Here and in the sequel, we use $\mathbf{x}^{(m)}$ to denote the monomial $\mathbf{x}_{1}^{m_{1}} \mathbf{x}_{2}^{m_{2}} \cdots \mathbf{x}_{n}^{m_{n}}$. The sum $\sum_{j=1}^{n} m_{j}$ is called the order of the moment. The time evolution of moments is given by the following result, which is a straightforward application of Theorem 1 in [12] to the SHS (4)-(6).

Theorem 1: For every continuously differentiable function $\psi: \mathbb{R}^{n} \rightarrow \mathbb{R}$ we have that

$$
\frac{d \mathbf{E}[\psi(\mathbf{x})]}{d t}=\mathbf{E}[(\mathbf{L} \psi)(\mathbf{x})]
$$

where $\forall \mathbf{x} \in \mathbb{R}^{n}$

$$
(\mathbf{L} \psi)(\mathbf{x})=\sum_{i=1}^{K}\left(\psi\left(\phi_{i}(\mathbf{x})\right)-\psi(\mathbf{x})\right) \lambda_{i}(\mathbf{x}) .
$$

The operator $\psi \mapsto \mathbf{L} \psi$ defined by (10) is called the extended generator of the SHS.

Since, the reset maps $\phi_{i}(\mathbf{x})$ and transitional intensities $\lambda_{i}(\mathbf{x})$ are finite polynomials ${ }^{2}$ in $\mathbf{x}$, the extended generator $(\mathbf{L} \psi)(\mathbf{x})$ is a finite-polynomial in $\mathbf{x}$ for every finite-polynomial $\psi(\mathbf{x})$ in $\mathbf{x}$. Such SHSs whose extended generator $L$ is closed on the set of finite-polynomials in $\mathbf{x}$ are called polynomial Stochastic Hybrid System ( $p S H S$ ).

Using (7), (9) and (10), the evolution of $\mu^{\left(m_{p}\right)}$ is given by

$$
\dot{\mu}^{\left(m_{p}\right)}=\mathbf{E}\left[\left(\mathbf{L} \psi^{\left(m_{p}\right)}\right)(\mathbf{x})\right]
$$

where

$$
\begin{aligned}
\left(\mathbf{L} \psi^{\left(m_{p}\right)}\right)(\mathbf{x}) & =\sum_{i=1}^{K} c_{i} h_{i}(\mathbf{x})\left\{\phi_{i}(\mathbf{x})^{\left(m_{p}\right)}-\mathbf{x}^{\left(m_{p}\right)}\right\} \\
& =\sum_{i=1}^{K} c_{i} h_{i}(\mathbf{x})\left\{\left[\prod_{j=1}^{n}\left(\mathbf{x}_{j}-u_{i j}+v_{i j}\right)^{m_{p_{j}}}\right]-\mathbf{x}^{\left(m_{p}\right)}\right\} .
\end{aligned}
$$

As discussed briefly in the previous section, the time derivative of moment of order $m^{*}$ is given by a linear combination of moments, moreover, because of (2), this linear combination of moments includes moments of order upto $m^{*}+1$. To see this, we re-write (11) as

$\dot{\mu}^{\left(m_{p}\right)}=\mathbf{E}\left[\sum_{i=1}^{K} c_{i} h_{i}(\mathbf{x}) \mathbf{x}^{\left(m_{p}\right)}\left\{\prod_{j=1}^{n}\left(1+\frac{v_{i j}-u_{i j}}{\mathbf{x}_{j}}\right)^{m_{p_{j}}}-1\right\}\right]$

where $\mu^{\left(m_{p}\right)}$ is a moment of order $m^{*}$. Now doing a binomial expansion, we have

$\dot{\mu}^{\left(m_{p}\right)}=\mathbf{E}\left[\sum_{i=1}^{K} c_{i} h_{i}(\mathbf{x}) \mathbf{x}^{\left(m_{p}\right)}\left(\sum_{j=1}^{n} \mathrm{C}_{1}^{m_{p_{j}}} \frac{v_{i j}-u_{i j}}{\mathbf{x}_{j}}+\ldots\right)\right]$.

\footnotetext{
${ }^{2}$ By a finite-polynomials in $x$ we mean a function $\lambda(x)$ such that $x \mapsto \lambda(x)$ is a (multi-variable) polynomial of finite degree.
}

With $\mathbf{x}^{\left(m_{p}\right)}$ being a monomial of order $m^{*}$, and for some reactions, $h_{i}(\mathbf{x})$ being a polynomial of order 2 , the polynomial

$$
\sum_{i=1}^{K} c_{i} h_{i}(\mathbf{x}) \mathbf{x}^{\left(m_{p}\right)} \sum_{j=1}^{n} \mathrm{C}_{1}^{m_{p_{j}}} \frac{v_{i j}-u_{i j}}{\mathbf{x}_{j}}
$$

will be order of $m^{*}+1$, and hence, will contribute moments of order $m^{*}+1$ in $\dot{\mu}^{\left(m_{p}\right)}$. Lesser order moments will appear from the other terms in the binomial expansion. Thus, if one now stacks all moments in an infinite vector $\mu_{\infty}$, its dynamics can be written as

$$
\dot{\mu}_{\infty}=A_{\infty} \mu_{\infty}
$$

for some appropriately defined infinite matrix $A_{\infty}$ [13]. In the sequel, we refer to (13) as the infinite-dimensional moment dynamics.

Since we are only interested in computing a few loworder moments, we rewrite (13) as

$$
\dot{\mu}=I_{k \times \infty} A_{\infty} \mu_{\infty}=A \mu+B \bar{\mu}, \quad \bar{\mu}=C \mu_{\infty},
$$

where $\mu \in \mathbb{R}^{k}$ contains to the top $k$ elements of $\mu_{\infty}$, which correspond to the lower-order moments of interest. $I_{k \times \infty}$ denotes a matrix composed of the first $k$ rows of the infinite identity matrix, $\bar{\mu} \in \mathbb{R}^{r}$ contains all the moments that appear in the first $k$ elements of $A_{\infty} \mu_{\infty}$ but that do not appear in $\mu$, and $C$ is the projection matrix that extracts $\bar{\mu}$ from $\mu_{\infty}$. Our goal is to approximate the infinite dimensional system (13) by a finite-dimensional nonlinear ODE of the form

$$
\dot{v}=A v+B \bar{\varphi}(v, t), \quad v=\left[v_{1}, v_{2}, \ldots, v_{n}\right]^{T}
$$

where the map $\bar{\varphi}: \mathbb{R}^{k} \times[0, \infty) \rightarrow \mathbb{R}^{r}$ should be chosen so as to keep $v(t)$ close to $\mu(t)$. We call (15) the truncated moment dynamics and $\bar{\varphi}$ the truncation function.

Let $\Omega_{\mu}$ be a set of initial conditions for which solutions to (13) exists globally and their first $k$ elements are uniformly bounded by the same constant. When a sufficiently large but finite number of derivatives of $\mu(t)$ and $v(t)$ match point-wise, then, the difference between solutions to (14) and (15) remains close on a given compact time interval, this follows from a Taylor series approximation argument. To be more precise, for each $\delta>0$ and $T \in \mathbb{R}$, there exists an integer $N$, sufficiently large, for which the following result holds: Assuming that for every $t_{0} \geq 0, \forall \mu_{\infty}\left(t_{0}\right) \in \Omega_{\mu}$

$$
\mu\left(t_{0}\right)=v\left(t_{0}\right) \Rightarrow \frac{d^{i} \mu\left(t_{0}\right)}{d t^{i}}=\frac{d^{i} v\left(t_{0}\right)}{d t^{i}}, \forall i \in\{1,2, \ldots, \mathrm{N}\}
$$

where $\frac{d^{i} \mu\left(t_{0}\right)}{d t^{i}}$ and $\frac{d^{i} v\left(t_{0}\right)}{d t^{i}}$ represent the $i^{t h}$ time derivative of $\mu(t)$ and $v(t)$ along the trajectories of system (13) and (15) respectively at $t=t_{0}$. Then,

$$
\|\mu(t)-v(t)\| \leq \delta, \forall t \in\left[t_{0}, T\right]
$$

along solutions of (13) and (15), where $\mu$ denote the first $k$ elements of $\mu_{\infty}$. It has been shown in [13] that under appropriate asymptotic stability conditions on (13), inequality (17) can actually be extended $\forall t \in\left[t_{0}, \infty\right)$. 


\section{Construction of Approximate Truncations}

In this section, using (16), we construct approximate truncated moment dynamics for the general set of reactions introduced in Section II. Since the quantities of interest in the stochastic approach are often the first and second order moments, we construct truncation models whose state contains all the first and second order moments of $\mathbf{x}$.

Let vector $\mu \in \mathbb{R}^{k}$ contain all the first and second order moments of $\mathbf{x}$. As with $n$ species, there are in all $^{3} 2 n+C_{2}^{n}$ first and second order moments, we have $k=2 n+C_{2}^{n}$. We denote the $p^{\text {th }}$ element of $\mu$ to be $\mu^{\left(m_{p}\right)}=\mathbf{E}\left[\psi^{\left(m_{p}\right)}(\mathbf{x})\right]$, $\psi^{\left(m_{p}\right)}(\mathbf{x})=\mathbf{x}^{\left(m_{p}\right)}$ where $m_{p}=\left(m_{p_{1}}, m_{p_{2}}, \ldots, m_{p_{n}}\right)$ and $\sum_{j=1}^{n} m_{p_{j}}=1$ or $2, \forall p=\{1, \ldots, k\}$. For convenience, we order the elements of $\mu$ in the following way.

1) The first $n$ elements are of the form $\mathbf{E}\left[\mathbf{x}_{j}\right]$, i.e, for each $p=\{1, \ldots, n\}, \quad m_{p}=e_{p}$ where $e_{p}=$ $\{0, \ldots, 0,1,0, \ldots, 0\}$ and the 1 occurs at the $p^{\text {th }}$ position.

2) The next $n$ elements are of the form $\mathbf{E}\left[\mathbf{x}_{j}^{2}\right]$, i.e, for each $p=\{n+1, \ldots, 2 n\}, m_{p}=2 e_{p-n}$.

3) The last $k-2 n$ elements are of the form $\mathbf{E}\left[\mathbf{x}_{j} \mathbf{x}_{t}\right], t \neq j$, i.e., for each $p=\{2 n+1, \ldots, k\}, m_{p}=e_{\bar{p}_{1}}+e_{\bar{p}_{2}}$ where $\bar{p}_{1}, \bar{p}_{2} \in\{1, \ldots, n\}, \bar{p}_{1} \neq \bar{p}_{2}$.

With this, the evolution of vector $\mu$ can be written as

$$
\dot{\mu}=A \mu+B \bar{\mu},
$$

for some matrices $A$ and $B$ obtained from (11) and as $\mu$ contains all the first and second order moments of $\mathbf{x}, \bar{\mu} \in \mathbb{R}^{r}$ is a vector of third order moments of $\mathbf{x}$ only. Our goal is to approximate (18) by a finite-dimensional nonlinear ODE of the form

$$
\dot{v}=A v+B \bar{\varphi}(v, t), \quad v=\left[v_{1}, v_{2}, \ldots, v_{k}\right]^{T}
$$

with $\bar{\varphi}: \mathbb{R}^{k} \times[0, \infty) \rightarrow \mathbb{R}^{r}$ chosen so that the condition (16) holds. For simplicity of computation, we restrict ourselves to functions $\bar{\varphi}(v(t))$, with entries $\varphi(v(t))$ of the following separable form

$$
\varphi(v(t))=\prod_{p=1}^{k} v_{p}(t)^{\gamma_{p}}=v(t)^{(\gamma)}, \quad \gamma=\left(\gamma_{1}, \ldots, \gamma_{k}\right)
$$

for appropriately chosen constants $\gamma_{p} \in \mathbb{R}$. In general, it is not possible to find $\bar{\varphi}(v(t)))$ of the form (20) for which (16) holds. We will therefore relax this condition and simply demand the following

$$
\mu\left(t_{0}\right)=v\left(t_{0}\right) \Rightarrow \frac{d^{i} \mu\left(t_{0}\right)}{d t^{i}}=\frac{d^{i} v\left(t_{0}\right)}{d t^{i}}+\mathbf{E}\left[\varepsilon_{i}\left(\mathbf{x}\left(t_{0}\right)\right)\right],
$$

$\forall i \in\{1,2\}$, where the $p^{\text {th }}$ element of $\mathbf{E}\left[\varepsilon_{i}\left(\mathbf{x}\left(t_{0}\right)\right)\right]$ contains moments of $\mathbf{x}\left(t_{0}\right)$. One can think of (21) as an approximation

$$
\begin{aligned}
& { }^{3} \mathrm{C}_{\hbar}^{\ell} \text { is defined as follows: } \forall \ell, \hbar \in \mathbb{N}_{\geq 0} \\
& \qquad \begin{array}{rlr}
\mathrm{C}_{\hbar}^{\ell} & =\frac{\ell !}{(\ell-\hbar) ! \hbar !}, \quad \ell \geq \hbar, \\
& =0, & \ell<\hbar .
\end{array}
\end{aligned}
$$

where $\ell$ ! denotes the factorial of $\ell$. to (16) which will be valid as long as the moments in $\frac{d^{i} \mu\left(t_{0}\right)}{d t^{i}}$ dominate $\mathbf{E}\left[\varepsilon_{i}\left(\mathbf{x}\left(t_{0}\right)\right)\right]$.

We make the following assumption on the stoichiometry of the reactions in (1), which is essential for the uniqueness for solution of $\gamma$.

Assumption 1 (Uniqueness): Let $\alpha_{i j}=v_{i j}-u_{i j}, \quad \forall i=$ $\{1, \ldots, K\}, \forall j=\{1, \ldots, n\}$. Then,

1) For each $j=\{1, \ldots, n\}$, there exists $q \in\{1, \ldots, S\}$ such that

$$
\sum_{i=K_{q-1}+1}^{K_{q}} c_{i} \alpha_{i j} \neq 0
$$

2) For each $p=\{2 n+1, \ldots, k\}$, there exists $q \in\{1, \ldots, S\}$ such that

$$
\sum_{i=K_{q-1}+1}^{K_{q}} c_{i} \alpha_{i \bar{p}_{1}} \alpha_{i \bar{p}_{2}} \neq 0
$$

The following theorem summarizes our result.

Theorem 2: For every deterministic initial condition, $\mathbf{x}\left(t_{0}\right)=x\left(t_{0}\right)$ with probability one, where $x\left(t_{0}\right)=$ $\left[x_{1}\left(t_{0}\right), \ldots, x_{n}\left(t_{0}\right)\right]^{T}$, there exists $\gamma$ for which

$$
\begin{aligned}
\mu\left(t_{0}\right)=v\left(t_{0}\right) & \Rightarrow \frac{d \mu\left(t_{0}\right)}{d t}=\frac{d v\left(t_{0}\right)}{d t} \\
& \Rightarrow \frac{d^{2} \mu\left(t_{0}\right)}{d t}=\frac{d^{2} v\left(t_{0}\right)}{d t}+\varepsilon_{2}\left(x\left(t_{0}\right)\right)
\end{aligned}
$$

where the first $n$ elements of $\varepsilon_{2}\left(x\left(t_{0}\right)\right)$ are zero and all other elements are polynomials in $x\left(t_{0}\right)$ of order 2 and $\frac{d^{i} \mu\left(t_{0}\right)}{d t^{i}}$, $\frac{d^{i} v\left(t_{0}\right)}{d t^{i}}$ represent the $i^{t h}$ time derivative of $\mu(t)$ and $v(t)$ along the trajectories of system (13) and (15), respectively at $t=t_{0}$. Furthermore, if Assumption 1 holds then $\gamma$ is uniquely determined.

Remark 1. As $\mu^{\left(m_{p}\right)}, \forall p \in\{n+1, \ldots, k\}$, is a second order moment, $\frac{d^{2} \mu^{\left(m_{p}\right)}}{d t^{2}}$ is a linear combination of moments of $\mathbf{x}$ upto order 4 . Thus, $\frac{d^{2} \mu^{(m p)}\left(t_{0}\right)}{d t^{2}}$ is a polynomial in $x\left(t_{0}\right)$ of order 4 and will generally dominate $\varepsilon_{2_{p}}\left(x\left(t_{0}\right)\right)$, the $p^{t h}$ element of $\varepsilon_{2}\left(x\left(t_{0}\right)\right)$, which is a polynomial in $x\left(t_{0}\right)$ of order 2. This would occur, e.g., with 10 molecules for all species, $\frac{d^{2} \mu^{\left(m_{p}\right)}\left(t_{0}\right)}{d t^{2}}$ would be atleast two orders of magnitude larger than $\varepsilon_{2_{p}}\left(x\left(t_{0}\right)\right)$.

Remark 2. If Assumption 1 holds, then, for each element $\mu^{(\bar{m})}$ of $\bar{\mu}$ given by $\bar{m}=\left(\bar{m}_{1}, \bar{m}_{2}, \ldots, \bar{m}_{n}\right), \sum_{j=1}^{n} \bar{m}_{j}=3, \gamma$ is uniquely determined by the following system of linear 
equations

$$
\begin{aligned}
& \bar{m}=\sum_{p=1}^{k} \gamma_{p} m_{p} \\
& \gamma_{p}=\left\{\begin{array}{l}
3 \text { if } \bar{m}_{p-n}=3 \\
1 \text { if } \bar{m}_{p-n}=2 \\
0 \text { if } \bar{m}_{p-n} \leq 1
\end{array} \quad \forall p \in\{n+1, \ldots, 2 n\}\right. \\
& \gamma_{p}=\bar{m}_{\bar{p}_{1}} \bar{m}_{\bar{p}_{2}}, \quad \forall p \in\{2 n+1, \ldots, k\} .
\end{aligned}
$$

As one see, the solution for $\gamma$ is independent of $K$, the stoichiometry of the reactions and the reaction rates. For $n=3$ and

$$
\begin{array}{r}
\mu=\left[\mu^{(1,0,0)}, \mu^{(0,1,0)}, \mu^{(0,0,1)}, \mu^{(2,0,0)}, \mu^{(0,2,0)}, \mu^{(0,0,2)},\right. \\
\left.\mu^{(1,1,0)}, \mu^{(0,1,1)}, \mu^{(1,0,1)}\right]^{T},
\end{array}
$$

truncation functions $\varphi(v)$ as given by (26)-(28), for various third order moments, , are as follows

$$
\begin{aligned}
& \mu^{(\bar{m})}=\mu^{(3,0,0)}, \quad \varphi(v)=\left(\frac{v_{4}}{v_{1}}\right)^{3} \\
& \mu^{(\bar{m})}=\mu^{(2,1,0)}, \quad \varphi(v)=\left(\frac{v_{4}}{v_{2}}\right)\left(\frac{v_{7}}{v_{1}}\right)^{2} \\
& \mu^{(\bar{m})}=\mu^{(1,1,1)}, \quad \varphi(v)=\left(\frac{v_{7}}{v_{1}}\right)\left(\frac{v_{8}}{v_{2}}\right)\left(\frac{v_{9}}{v_{3}}\right) .
\end{aligned}
$$

One can verify that the approximation of higher order moments with lower order ones, as given by the truncation functions above, is consistent with $\mathbf{x}(t)$ being lognormally distributed.

\section{EXAMPLE}

Consider the following decaying-dimerizing reaction set [7], [14]:

$$
X_{1} \stackrel{c_{1}=1}{\longrightarrow} *, \quad 2 X_{1} \stackrel{c_{2}=10}{\longrightarrow} X_{2}, \quad X_{2} \stackrel{c_{3}=1000}{\longrightarrow} 2 X_{1}, \quad X_{2} \stackrel{c_{4}=.1}{\longrightarrow} * .
$$

The number of particles $\mathbf{x}=\left[\mathbf{x}_{1}, \mathbf{x}_{2}\right]^{T}$ of the species involved in the above set of decaying-dimerizing reaction can be generated by a SHS with continuous dynamics $\dot{\mathbf{x}}=0$ and four reset maps

$$
\begin{aligned}
& \mathbf{x} \mapsto \phi_{1}(\mathbf{x})=\left[\begin{array}{c}
\mathbf{x}_{1}-1 \\
\mathbf{x}_{2}
\end{array}\right], \lambda_{1}(\mathbf{x})=c_{1} \mathbf{x}_{1} \\
& \mathbf{x} \mapsto \phi_{2}(\mathbf{x})=\left[\begin{array}{c}
\mathbf{x}_{1}-2 \\
\mathbf{x}_{2}+1
\end{array}\right], \lambda_{2}(\mathbf{x})=\frac{1}{2} c_{2} \mathbf{x}_{1}\left(\mathbf{x}_{1}-1\right) \\
& \mathbf{x} \mapsto \phi_{3}(\mathbf{x})=\left[\begin{array}{c}
\mathbf{x}_{1}+2 \\
\mathbf{x}_{2}-1
\end{array}\right], \lambda_{3}(\mathbf{x})=c_{3} \mathbf{x}_{2} \\
& \mathbf{x} \mapsto \phi_{4}(\mathbf{x})=\left[\begin{array}{c}
\mathbf{x}_{1} \\
\mathbf{x}_{2}-1
\end{array}\right], \lambda_{4}(\mathbf{x})=c_{4} \mathbf{x}_{2} .
\end{aligned}
$$

Taking

$$
\mu=\left[\begin{array}{c}
\mu^{(1,0)} \\
\mu^{(0,1)} \\
\mu^{(2,0)} \\
\mu^{(0,2)} \\
\mu^{(1,1)}
\end{array}\right]
$$

and with reset maps and transitional intensities as given above, from (11) the evolution of $\mu$ is given by

$$
\dot{\mu}=A \mu+B \bar{\mu} \text {. }
$$

where

$$
\begin{aligned}
& A=\left[\begin{array}{rcccc}
9 & 2000 & -10 & 0 & 0 \\
-5 & -1000.1 & 5 & 0 & 0 \\
-19 & 4000 & 38 & 0 & 4000 \\
-5 & 1000.1 & 5 & -2000.2 & -10 \\
10 & -2000 & -15 & 2000 & -991.1
\end{array}\right] \\
& B=\left[\begin{array}{rc}
0 & 0 \\
0 & 0 \\
-20 & 0 \\
0 & 10 \\
5 & -10
\end{array}\right], \quad \bar{\mu}=\left[\begin{array}{l}
\mu^{(3,0)} \\
\mu^{(2,1)}
\end{array}\right] .
\end{aligned}
$$

One can verify that the stoichiometry of the above reaction satisfies Assumption 1, and hence, from Theorem 2, there exists a unique $\gamma$ such that (24) and (25) hold. Using (29) and (30) the truncated moment dynamics is given by

$$
\dot{v}=A v+B \bar{\varphi}(v), \quad \bar{\varphi}(v)=\left[\begin{array}{c}
\left(\frac{v_{3}}{v_{1}}\right)^{3} \\
\left(\frac{v_{3}}{v_{2}}\right)\left(\frac{v_{5}}{v_{1}}\right)^{2}
\end{array}\right] .
$$
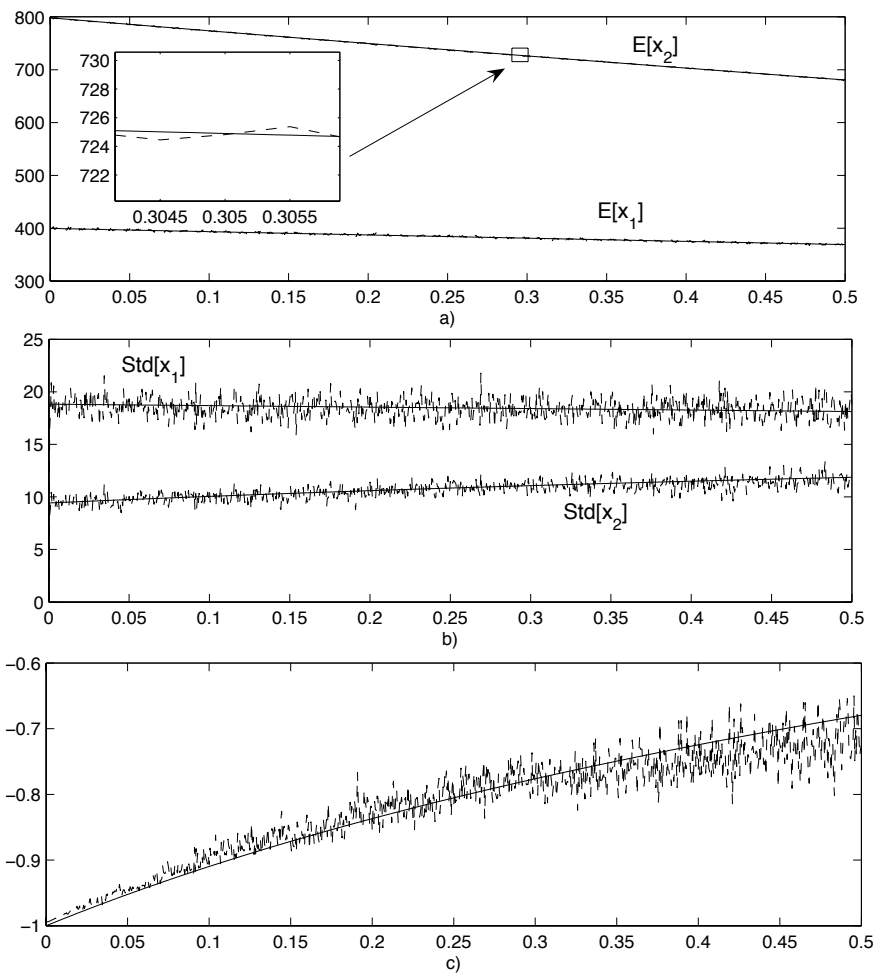

Fig. 1. Comparison between Monte Carlo simulations (dashed lines) and the truncated model (33) (solid lines), for the evolution of a) the means, b) the standard deviations and c) the correlation $\frac{\mathbf{E}\left[\mathbf{x}_{1} \mathbf{x}_{2}\right]-\mathbf{E}\left[\mathbf{x}_{1}\right] \mathbf{E}\left[\mathbf{x}_{2}\right]}{\operatorname{Std}\left[\mathbf{x}_{1} \mid \operatorname{Std}\left[\mathbf{x}_{2}\right]\right.}$ with initial conditions (34).

The particular values of the parameter chosen in (32) result in two distinct time scales, which makes this chemical reaction computationally difficult to simulate ("stiff" in the terminology of [14]). Figure 1 shows a comparison between 

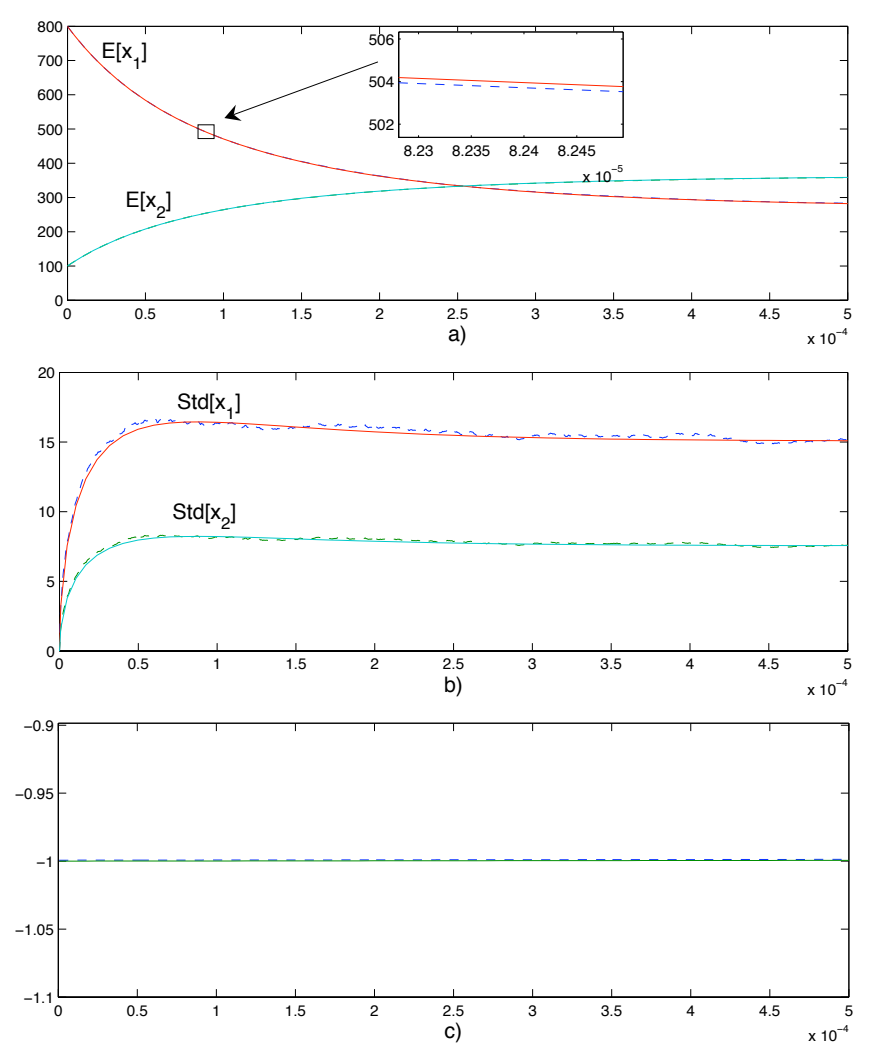

Fig. 2. Comparison between Monte Carlo simulations (dashed lines) and the truncated model (33) (solid lines), for the evolution of a) the means, b) the standard deviations and c) the correlation $\frac{\mathbf{E}\left[\mathbf{x}_{1} \mathbf{x}_{2}\right]-\mathbf{E}\left[\mathbf{x}_{1}\right] \mathbf{E}\left[\mathbf{x}_{2}\right]}{\operatorname{Std}\left[\mathbf{x}_{1}\right] \operatorname{Std}\left[\mathbf{x}_{2}\right]}$ with initial conditions (35).

Monte Carlo simulations and the truncated model (33) for initial conditions

$$
\mathbf{x}_{1}(0)=400, \quad \mathbf{x}_{2}(0)=798 .
$$

Monte Carlo simulations were carried out using the algorithm described in [12], which is equivalent to Gillespie's Monte Carlo simulation (SSA) for chemical reactions [6]. The above initial conditions (34) start in the "slow manifold" $\mathbf{x}_{2}=$ $\frac{5}{1000} \mathbf{x}_{1}\left(\mathbf{x}_{1}-1\right)$ and Figure 1 essentially shows the evolution of the system on this manifold. Figure 2 zooms in on the interval $\left[0,5 \times 10^{-4}\right]$ and shows the evolution of the system towards the manifold when it starts away from it at

$$
\mathbf{x}_{1}(0)=800, \quad \mathbf{x}_{2}(0)=100 .
$$

As one can see from the zoomed inserts in Figure 1 and Figure 2, the truncated model (33) captures both the time scales and provides very accurate estimate for the evolution of the first and second order moments of $\mathbf{x}_{1}$ and $\mathbf{x}_{2}$.

\section{CONCLusion And Future Work}

An approximate stochastic model for chemically reacting systems was presented in this paper. This was done by representing the population of various species involved in a set of chemical reactions as the continuous state of a pSHS. With such a representation, the dynamics of the infinite vector containing all the statistical moments of the continuous state are governed by an infinite-dimensional linear system of ODEs, which under appropriate conditions can be approximated by finite-dimensional nonlinear ODEs. For a very general class of chemical reactions, explicit conditions under which these finite-dimensional nonlinear ODEs exist and are unique were given along with formulas to construct them. Using these formulas, we constructed an approximate stochastic model for the decaying-dimerizing reaction set, which provided very accurate results in comparison with Monte Carlo algorithms.

An important observation which points to directions for future research is as follows: If one restricts the class of reactions in (1) to only single specie reactions, and if (22) holds for $n=1$, then, with $\gamma$ chosen as solution of (26) and (27), equality (21) holds $\forall i \geq 2$. We believe that a similar result can be proven for multi-specie reactions.

\section{ACKNOWLEDGMENTS}

We would like to thank Mustafa Khammash and Hana ElSamad for several discussions that led us to consider pSHS as a modeling tool for chemical reactions.

\section{REFERENCES}

[1] H. H. McAdams and A. P. Arkin, "Stochastic mechanisms in gene expression," Proceedings of the National Academy of Sciences U.S.A, vol. 94, pp. 814-819, 1997.

[2] A. Arkin, J. Ross, and H. H. McAdams, "Stochastic kinetic analysis of developmental pathway bifurcation in phage $\lambda$-infected Escherichia coli cells," Genetics, vol. 149, pp. 1633-1648, 1998.

[3] D. Endy and R. Brent, "Modelling cellular behaviour," Nature (London), vol. 409, pp. 391-395, 2001.

[4] J. Hasty, J. Pradines, M. Dolnik, and J. J. Collins, "Noise-based switches and amplifiers for gene expression," Proceedings of the National Academy of Sciences U.S.A, vol. 97, pp. 2075-2080, 2000.

[5] N. G. V. Kampen, Stochastic Processes in Physics and Chemistry. Amsterdam, The Netherlands: Elsevier Science, 2001.

[6] D. T. Gillespie, "A general method for numerically simulating the stochastic time evolution of coupled chemical reactions," J. of Computational Physics, vol. 22, pp. 403-434, 1976.

[7] D. T. Gillespie and L. R. Petzold, "Improved leap-size selection for accelerated stochastic simulation," J. of Chemical Physics, vol. 119, no. 16 , pp. $8229-8234$, Oct. 2003.

[8] M. A. Gibson and J. Bruck, "Efficient exact stochastic simulation of chemical systems with many species and many channels," $J$. of Physical Chemistry A, vol. 104, pp. 1876-1889, 2000.

[9] D. T. Gillespie, "Approximate accelerated stochastic simulation of chemically reacting systems," J. of Chemical Physics, vol. 115, no. 4, pp. 1716-1733, 2001.

[10] J. P. Hespanha and A. Singh, "Stochastic models for chemically reacting systems using polynomial stochastic hybrid systems," Int. Journal of Robust and Nonlinear Control, 2005. To appear.

[11] F. Wilkinson, Chemical Kinetics and Reaction Mechanisms. New York: Van Nostrand Reinhold Co, 1980.

[12] J. P. Hespanha, "Stochastic hybrid systems: Applications to communication networks," in Hybrid Systems: Computation and Control, ser. Lect. Notes in Comput. Science, R. Alur and G. J. Pappas, Eds. Berlin: Springer-Verlag, Mar. 2004, no. 2993, pp. 387-401.

[13] — , "Polynomial stochastic hybrid systems," in Hybrid Systems : Computation and Control (HSCC) 2005, Zurich, Switzerland, 2005.

[14] M. Rathinam, L. R. Petzold, Y. Cao, and D. T. Gillespie, "Stiffness in stochastic chemically reacting systems: The implicit tau-leaping method," J. of Chemical Physics, vol. 119, no. 24, pp. 12 784-12 794, Dec. 2003. 